\title{
Real-Time Group Face-Detection for an Intelligent Class-Attendance System
}

\author{
Abdelfatah Aref Tamimi \\ Faculty of Sciences \& IT, Al-Zaytoonah University of Jordan, Dept. of Computer Science, P.O. Box130, Amman \\ (11733), Jordan \\ E-mail: drtamimi@zuj.edu.jo
}

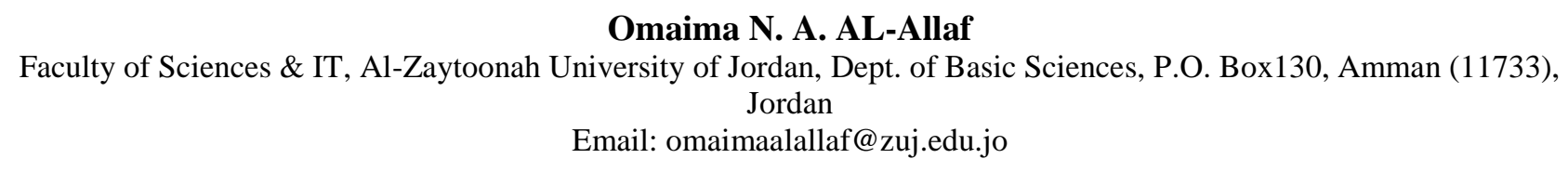

Mohammad A. Alia

Faculty of Sciences \& IT, Al-Zaytoonah University of Jordan, Dept. of Computer Information Systems, P.O. Box130, Amman (11733), Jordan

Email: dr.m.alia@zuj.edu.jo

\begin{abstract}
The traditional manual attendance system wastes time over students' responses, but it has worked well for small numbers of students. This research presents a real-time group face-detection system. This system will be used later for student class attendance through automatic student identification. The system architecture and its algorithm will be described in details. The algorithm for the system was based on analyzing facial properties and features in order to perform face detection for checking students' attendance in real time. The classroom's camera captures the students' photo. Then, face detection will be implemented automatically to generate a list of detected student faces. Many experiments were adopted based on real time video captured using digital cameras. The experimental results showed that our approach of face detection offers realtime processing speed with good acceptable detection ratio equal to $94.73 \%$.
\end{abstract}

Index Terms - Face Detection, Group Face Detection, RealTime Face Detection, Attendance System

\section{INTRODUCTION}

Humans are good in identifying known faces, but they are not as good when they deal with a large number of unknown faces. It is a great challenge to build software which is similar to human ability in detecting and recognizing faces in real time. Nevertheless, it is very important to use automated attendance systems, especially in student classrooms of universities where a good face recognition system executed on a fast computer with a large storage capacity can overcome human limitations. Generally, face recognition remains a demanded technology [1].

The human face presents several challenges for any visual system, such as the high degree of similarity between different faces, the extent to which expressions and hair can alter the face, and the large number of angles from which a face can be viewed in common situations.
A face recognition system must be robust with respect to this variability and be generalized over a wide range of conditions to capture the essential similarities for a given human face [1]. A general face recognition system consists of many stages: face detection; feature extraction and face recognition [2].

The face detection and feature extraction phases could be performed concurrently. The system identifies a certain image region as a face. This procedure has many applications: face tracking, pose estimation, or compression. Feature extraction involves obtaining relevant facial features from the data. These features could be certain face regions, variations, angles, or measures. This phase has other applications such as facial feature tracking and emotion recognition. Finally, the system recognizes the face by reporting an identity from a database. Face detection and recognition can be applied in many fields such as authentication, security, video surveillance, and human interaction systems [3]. Faces represent complex, multidimensional, meaningful visual stimuli and developing a computational model for face recognition is difficult [4]. As mentioned earlier, the task of detecting a face is complex due to its variability including its pose, color, expression, orientation, and position. Therefore, recognizing various facial expressions is applied by using various modeling techniques [5][33][34].

Recently, applications based on human face detection have been developed for a variety of hardware and software systems such as surveillance systems, digital monitoring, intelligent robots, laptops, PC cameras, digital cameras, and $3 \mathrm{G}$ cell phones. These applications play an important role in our life. Nevertheless, the algorithms of most applications are complicated and hard to meet real-time requirements of specific frame-rates [6].

Therefore, over the past decade, many approaches for improving the performance of face detection have been 
proposed [6..10]. Other literature studies focused on the survey of face detection techniques [11..15]. The importance of the automatic face detection and tracking system has increased as it is needed for video surveillance and new user interfaces [11]. At the same time, few literature studies related to real time face detection [16..22]. Therefore, general architecture and analytical steps were suggested in our previous work for the system of monitoring and recognizing students during class [23]. This current work includes a detailed description of an algorithm related to the real-time group face-detection step of our previous work [23]. The rest of this study is organized as follows: Section 2 includes the literature studies related to real time face detection. Whereas, Section 3 gives a full description of the suggested system including system input, output, main steps, and mathematical models used to detect faces with the best front position. Section 4 includes the experiments and finally Section 5 concludes this work.

\section{II.LITERATURE STUDIES RELATED TO REAL TIME FACE DETECTION}

Face recognition systems ranged from static to dynamic uncontrolled face identification in cluttered background (subway and airport). Face recognition can be regarded as a set of common biometric properties used by humans to recognize persons [24]. Face verification extracts a feature set from a two-dimensional face image and matches it with a template stored in a database [25]. Facial images may be captured in unconstrained and uncontrolled environments. Therefore, the recognition performance of the existing face recognition techniques is low. This is because of appearance variations of the face image induced by different environmental factors such as illumination. Changes in illumination conditions cause variation in face appearance and affect face recognition systems performance [26]. The face recognition phase includes a comparison method, a classification algorithm, and an accuracy measure. This recognition phase uses data mining methods for classification [14]. Prakash et al., 2013 [27] provided a description of single feature and multiple features depending on biometric systems by including the performance of physiological specifications (such as fingerprint, iris, and face recognition) and behavioral specifications (such as body language, facial expression, and speech recognition).

Face detection is difficult because of the textural differences among faces. The great challenge for the face detection is the large number of factors [13] such as: pose, orientation, facial expressions, facial size, luminance conditions, occlusion, structural components, gender, lighting conditions, the scene, and the complexity of image background (which ranges from simple uniform to complex backgrounds). At the same time, faces closer to the camera appear larger than faces that are farther away from the camera. Face detection is the first step in online face recognition. Face detection and localization is the task of checking whether the given input image contains a human face and returning the location of this face in the image.

In recent years, there have been a wide variety of applications and researches related to real-time face detection [24]. Rob McCread, 2000 [16] developed an object detection system that operates on real-time video data in hardware for human faces. This system was implemented on a large programmable hardware system to reduce the time and cost required to create a working hardware prototype. The implementation runs at 30 frames per second, which is 1000 times faster than the same algorithm running on software.

Paul and Michael, 2001 [17] described a visual object detection framework to process images to achieve high detection rates. While Shakhnarovich et al., 2002 [18] presented progress toward a real-time face detection and a demographic analysis system. Faces are detected and extracted using the algorithm proposed in [17]. Detected faces are passed to a demographics classifier which uses the face detector architecture. This classifier is fast and delivers error rates better than other known classifiers. The entire system processes 10 frames per second on an $800 \mathrm{MHz}$ Intel PIII.

After that, Paul and Michael, 2004 [19] described a face detection framework to process images by achieving high detection rates. They introduced a new image representation, "Integral Image," to allow features used by the detector to be computed quickly. They used a simple classifier to select a small number of visual features from a large set of potential features. They introduced a method for combining classifiers in a cascade to allow background regions of the image to be quickly discarded while spending more computation on face-like regions. The system yields face detection that proceeds at 15 frames per second.

Smart Cameras are becoming more popular in the intelligent surveillance systems area to recognize faces in a crowd in real-time to enhance these systems. Using a high resolution smart camera as a tool to extract faces that are suitable for face recognition would reduce the computational load on the main processing unit. This processing unit would not be overloaded by high data rates required for high resolution video and could be designed solely for face recognition [20]. Mustafah et al., 2007 [20] reported on a multiple-stage face detection and tracking system that is designed for implementation on the NICTA high resolution (5 MP) smart camera. Whereas, Zhiwei and Qiang, 2005 [21] proposed a realtime method for eye tracking under variable lighting conditions and face orientations based on combining the latest technologies in appearance-based object recognition. Combining the strengths of different techniques and overcoming their shortcomings, the proposed method uses an active infrared illumination to brighten the subject's face to produce the bright pupil effect. The bright pupil effect and the appearance of eyes are utilized simultaneously for eyes detection and tracking.

Finally, Michalis, 2013 [22] suggested a framework to track and identify many persons in a crowded location 
captured by multiple cameras. A people detector is employed to estimate the position of individuals. These position estimates are used by the face detector to reduce the search space of possible face locations and minimize the false positives.

The goal of this research is to construct an automatic face detection system using a PC camera in real-time. This system will be used later for student class attendance. The focus of this research is on the face detection stage of face recognition.

\section{PROPOSED SYSTEM}

In our previous work [23], a novel system for recognizing students during a class session is described. This system includes the following steps: system access control to authenticate the instructor in the student database server; face detection and recognition (after capturing the students' photos' by camera); and collecting data process and producing the attendance report. This section includes a detailed description of the algorithm suggested for the face detection step in the proposed system.

\section{A. System Input}

The input of real time group face detection system is a set of real time video captured photos (frames). Each frame is taken for a group of students which attend any class as shown in Fig.1. Whereas the output of this system is a set of individual photos related to acceptable detected faces in the captured photos, one photo for each student per frame.

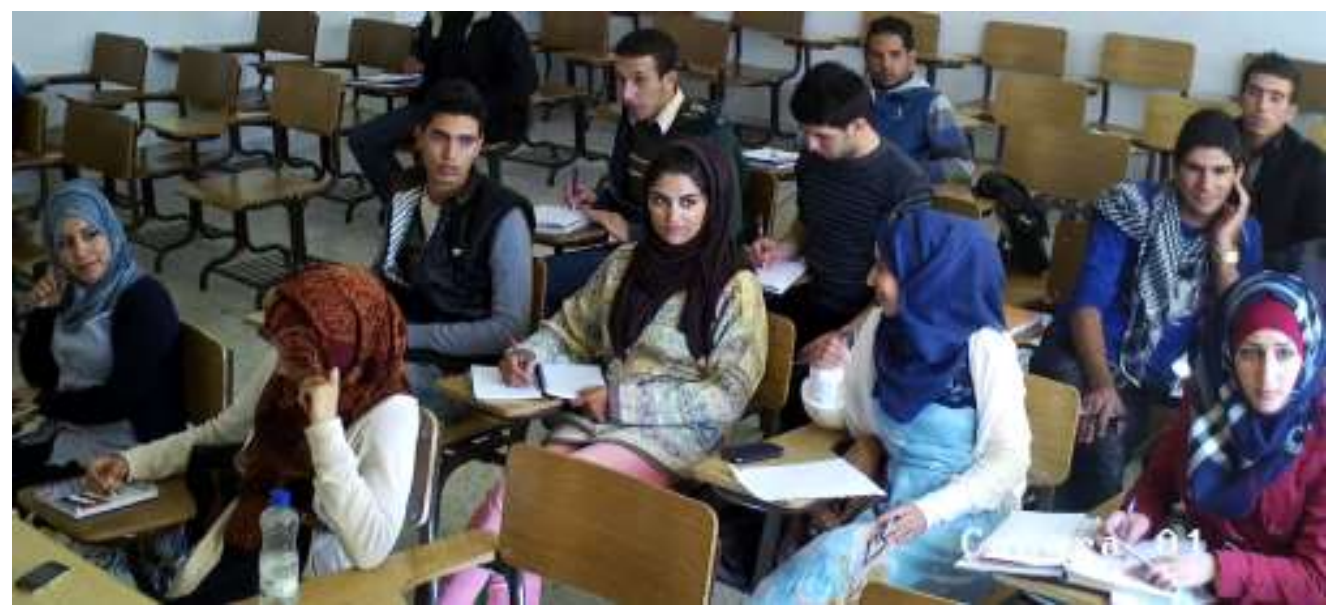

Fig. 1. Photo of group of 12 students in a class

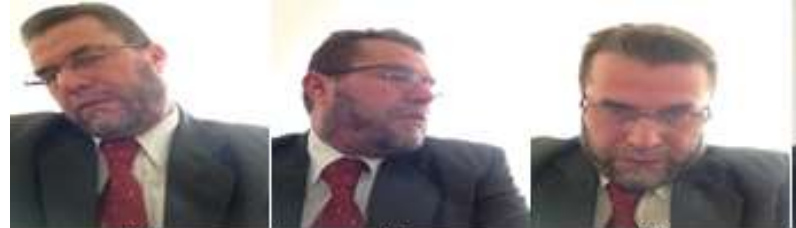

(a)

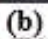

(b)

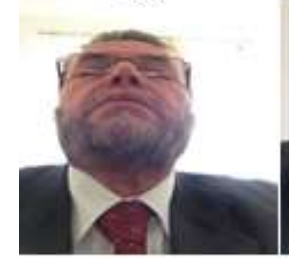

(f)

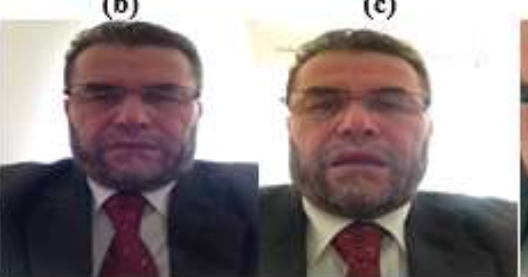

(g) (h)

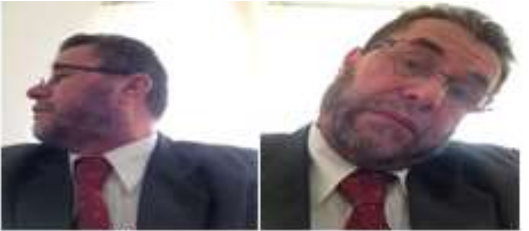

(d)

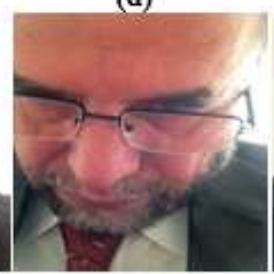

(i) (e)

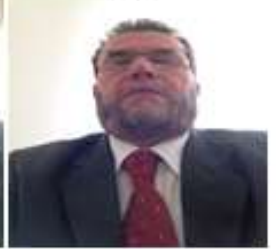

(j)

Fig. 2. The Photo with best direction position of face will be selected

\section{B. Main Steps of the System}

The main steps of the proposed system are as follows:

1. The number of frames for the overall class will be determined earlier in the program to avoid infinite loop. We will be assumed that acceptable results can be obtained when the number of frames increased.

2. The number of photos (faces) to be stored in a system database depends on the number of students that already registered in the class. This number will affect the maximum number of faces to be detected in each camera shot.

3. The number of faces to be detected in each camera shut photo will be determined earlier. It is equal to the maximum number of students which registered in the class.

4. Each camera photo includes all students' faces. All well detected faces will be chosen according to mathematical suggested formula as we will show later in the next section. 
5. Continue taking frames until all faces are detected with acceptable ratio or the maximum number of frames reached.

6. For example, Fig. 2 shows 10 faces of one person taken when running the algorithm for 10 frames.

7. Our algorithm choose the acceptable photo (Fig.2.g) for detection of face (left eye, right eye, nose and mouth) as shown in Fig.3 which will be stored to be used for face recognition. At this step, only well detected face photo (face with best front position) will be used for face recognition.

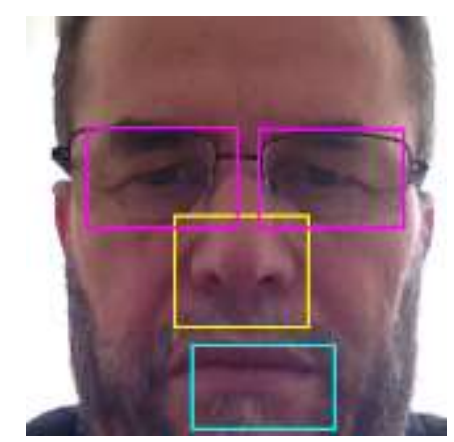

Fig. 3. Four Face Regions.

\section{C.Mathematical Models Used to Detect Faces with Best Front Position}

For each face photo, relations between the four face regions (left eye, right eye, nose and mouth) are calculated to find the best direction position of face. This is done according to suggested algorithm as follows:

a) Each detected face in camera shoot photo is divided into 4 regions (two eyes, nose and mouth) as shown in Fig.3. Using Computer Vision System Toolbox from MathLab 2012a.

b) Find the center points of all boxes (left eye, right eye, nose and mouth), name them as P1, P2, P3 and P4 consequently as shown in Fig.4.

c) Find the distances (a1, a2, a3, b2 and b3) between the four points (P1, P2, P3 and P4) as shown in Fig.4 by using (1) derived from Pythagorean theorem [28..30].

$$
d=\sqrt{(x-x 1)^{2}+(y-y 1)^{2}}
$$

d) Calculate the triangle areas for points P1, P2 and P3 and also for points: P1, P2 and P4 according to Heron's formulas (2) and (3) [30][31] as follows:

$$
\begin{aligned}
& \mathrm{s}=\frac{\mathrm{a}+\mathrm{b}+\mathrm{c}}{2} \\
& \text { area }=\sqrt{\mathrm{s}(\mathrm{s}-\mathrm{a})(s-b)(s-c)}
\end{aligned}
$$

Where $\mathrm{a}, \mathrm{b}$ and $\mathrm{c}$ are triangle sides respectively.

e) Calculate the ratio $\left(A_{i}\right)$ between two areas using (4) to obtain best results to find best direction position of face.

$$
A_{i}=\frac{\text { Area of Big Triangle }}{\text { Area of small Triangle }}
$$

f) Calculate the angels $(\Theta 1, \Theta 2, \Theta 3, \beta 1, \beta 2$ and $\beta 3)$, by using Harley's law of Cosine (5) [32]:

$$
\cos ^{-1} \theta=\frac{c^{2}-a^{2}-b^{2}}{2 a b}
$$

g) If the two angles $(\Theta 1$ and $\Theta 2)$ of the small triangle is within an acceptable threshold value (T1) as shown in (6). Also if the differences between the two angles $\beta 1$ and $\beta 2$ is within an acceptable threshold value (T2) as shown in (7). Many experiments will be conducted with different values of $\mathrm{T} 1, \mathrm{~T} 2$ and $\mathrm{Ai}$

$$
£ 1=|\Theta 1-\Theta 2| \leq \mathrm{T} 1
$$

Where, $\Theta 1$ is left eyes nose angles, and $\Theta 2$ is right eyes nose angles

$$
£ 2=|\beta 1-\beta 2| \leq \mathrm{T} 2
$$

Where, $\beta 1$ is left eyes mouth angles, and $\beta 2$ is right eyes mouth angles.

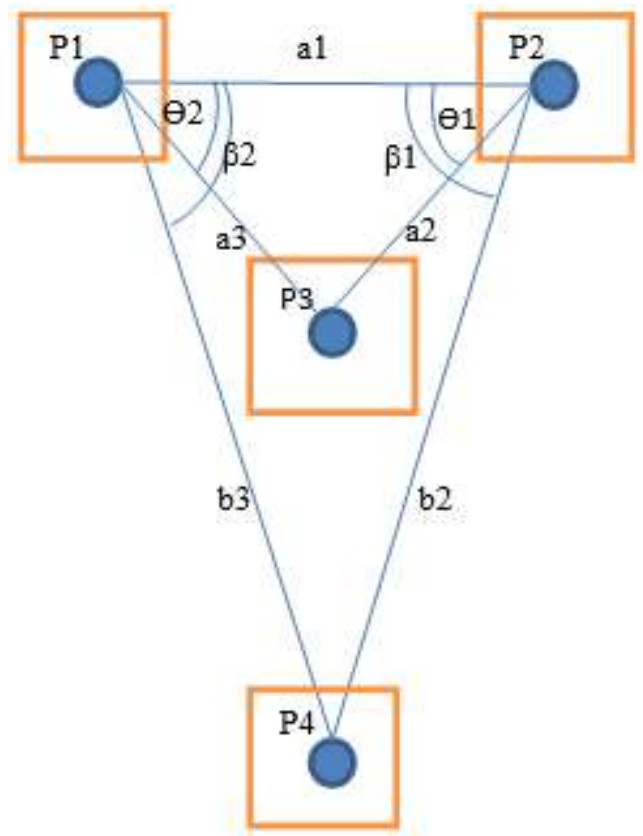

Fig. 4. The Distances between face regions

\section{D.System Algorithm}

Step1: initialize the maximum number of frames $(\mathrm{F})$ and maximum number of students in the class (ST).

Step2: read one frame (which has student's photos).

Step3: search for faces of all students in the frame.

Step4: if face with best front position find (according to T1 and T2 thresholds, formulas (6) and (7)), store face in student database.

Step5: repeat until students' faces are detected or maximum number of frames reached.

\section{EXPERIMENTS}

The real time group face detection system is implemented using MathLib. The program steps are described in previous section. Step 5.a is implemented using "detectFaceParts" method from Computer Vision System Toolbox and Image Processing Toolbox in MathLib 2012a. 
Many experiments were conducted for this study. In first experiment, 10 camera shoot photos (frames) are taken for a group of 12 students in lecture1. The 12 Photos with best direction position of faces were stored in the system database. The best 12 face photos were selected according to (1) to (7) by setting the values of T1 and T2 to 5. Table 1 shows the values of $\mathrm{A}, \Theta 1, \Theta 2, \beta 1$, $\beta 2, £_{1}$ and $£_{2}$ of the 12 face photos.

The values of $A_{i}$ related to each face were calculate. We can notices from Table 1 that the average values of parameter (A) are around 1.7.

Table 1. The values of $A, \Theta 1, \Theta 2, \beta 1$, and $\beta 2$ of the 12 face photos

\begin{tabular}{|c|c|c|c|c|c|c|c|c|}
\hline student & $\mathrm{Ai}$ & $\theta 1_{\mathrm{i}}$ & $\theta 2_{i}$ & $£_{1}(\Theta 2-\Theta 1)$ & $\beta 1_{\mathrm{i}}$ & $\beta 2_{\mathrm{i}}$ & $£_{2}(\beta 2-\beta 1)$ & $£_{1}-£_{2}$ \\
\hline 1 & 1.540385 & 51.52881 & 58.18409 & 6.65528 & 64.81432 & 65.85048 & 1.03616 & 5.61912 \\
\hline 2 & 1.690715 & 59.41835 & 46.77834 & 12.64001 & 65.66185 & 65.62656 & 0.03529 & 12.60472 \\
\hline 3 & 2.008267 & 42.97134 & 53.4652 & 10.49386 & 64.34403 & 67.06593 & 2.7219 & 7.77196 \\
\hline 4 & 1.697877 & 51.20139 & 49.09815 & 2.10324 & 57.49714 & 70.88385 & 13.38671 & 11.28347 \\
\hline 5 & 1.920368 & 43.96973 & 51.72928 & 7.75955 & 61.62187 & 67.68577 & 6.0639 & 1.69565 \\
\hline 6 & 1.592346 & 64.53203 & 48.3783 & 16.15373 & 66.63182 & 66.97324 & 0.34142 & 15.81231 \\
\hline 7 & 1.664077 & 41.67697 & 55.60689 & 13.92992 & 58.51456 & 64.64233 & 6.12777 & 7.80215 \\
\hline 8 & 1.800216 & 70.80263 & 52.89694 & 17.90569 & 73.58446 & 72.30929 & 1.27517 & 16.63052 \\
\hline 9 & 1.811259 & 47.01162 & 50.2753 & 3.26368 & 60.97764 & 67.28672 & 6.30908 & 3.0454 \\
\hline 10 & 1.762203 & 53.36795 & 44.44419 & 8.92376 & 66.80952 & 60.22528 & 6.58424 & 2.33952 \\
\hline 11 & 1.790071 & 51.47321 & 46.90088 & 4.57233 & 65.41189 & 62.98074 & 2.43115 & 2.14118 \\
\hline 12 & 1.734421 & 45.90664 & 64.4641 & 18.55746 & 68.39321 & 66.34749 & 2.04572 & 16.51174 \\
\hline
\end{tabular}

At the same time, the values of $£ 1$ and $£ 2$ were calculated to determine if the face is detected or not within accepted numbers $\mathrm{T} 1$ and $\mathrm{T} 2$.

If one or more of the following attributes are within the acceptable threshold $\left(A_{i}, £ 1\right.$ and $\left.£ 2\right)$ where $A_{i}, £ 1$ and $£ 2$ as shown previously in (3), (4) and (5) respectively.

Table 1 shows also that most values of $£ 1$ (difference between left eyes nose angles and right eyes nose angles) are not greater than T1(5). At the same time, many values of $£ 2$ (left eyes mouth angles and right eyes mouth angles) are not greater than $\mathrm{T} 2(5)$.

A threshold value $<=13$ was suggested and compared with abs $(£ 1-£ 1)$ to determine if the face is detected or not. Table 2 shows exactly which face is acceptable detected or not. The accepted faces will be stored in a data base and unaccepted faces will be searched for in the next frame.

We can note from Table 2 which is related to experiment 1 that only 3 faces are not acceptable and other 9 faces are acceptable. Then these 9 acceptable faces will be stored in database folder 1 which is related to lecture 1.

Other experiments (2..10) were conducted for lecture2 to lecture 10 each with different number of students. For each experiment (class lecture), 10 frames will be taken to detect faces and stored only an acceptable detected faces in a database folder for this lecture. These acceptable detected faces will be used later in face recognition step for student class attendance system.

Finally the detected accepted ratio ( $r$ ) is calculated for each lecture experiment as shown in (8). Table 3 shows exactly the acceptable ratio for each experiment.

\section{$r=\frac{\text { number of detected faces }}{\text { number of total faces in class }}$}

We can note from Table 3 that the detected accepted ratio is ranged between $75 \%$ and $94.73 \%$.

Table 3. The acceptable ratio for each experiment

\begin{tabular}{|c|c|c|c|}
\hline Lecture & $\begin{array}{c}\text { Number } \\
\text { of students }\end{array}$ & $\begin{array}{c}\text { Detected } \\
\text { faces }\end{array}$ & $\begin{array}{c}\text { Accepted } \\
\text { ratio. }\end{array}$ \\
\hline lecture 1 & 12 & 9 & 75 \\
\hline lecture 2 & 38 & 36 & 94.73 \\
\hline lecture 3 & 38 & 34 & 89.47 \\
\hline lecture 4 & 40 & 37 & 92.50 \\
\hline lecture 5 & 25 & 22 & 88 \\
\hline lecture 6 & 20 & 17 & 85 \\
\hline lecture 7 & 30 & 27 & 90 \\
\hline lecture 8 & 35 & 32 & 91.42 \\
\hline lecture 9 & 20 & 18 & 90 \\
\hline lecture 10 & 35 & 33 & 94.28 \\
\hline
\end{tabular}

\section{CONCLUSION}

In this paper, we have proposed a group face detection algorithm to detect student faces during any class lecture. This algorithm works in real time and depends on catching the video from a PC digital camera. This algorithm was implemented using MathLib 2012.

Many experiments were conducted in 10 different class lectures with different numbers of students. This is done to evaluate the quality (acceptance ratio) of the suggested 
algorithm that detects student faces in any image frame in a captured video. The experimental results showed that the acceptance detection ratio of our suggested algorithm ranged from $75 \%$ to $94.73 \%$.

In our planned future work, these accepted detected faces for each class lecture will be stored in its own folder to build a full database which includes many folders for each lecture.
These accepted detected faces will be used later in the recognition step of the student class attendance system. Our next future work is to suggest a new algorithm for the recognition step. The recognition step will depend on the suggested algorithm of the current work.

Table 2. Shows the accepted and not accepted faces according to Ai, £1 and $£ 2$

\begin{tabular}{|c|c|c|c|c|c|c|}
\hline $\begin{array}{l}\text { Student } \\
\text { Number }\end{array}$ & Student Face & $\mathrm{Ai}$ & $£ 1$ & $£ 2$ & $\operatorname{abs}\left(£_{1}-£_{2}\right)$ & $\begin{array}{l}\text { Accepted and not } \\
\text { accepted faces }\end{array}$ \\
\hline 1 & & 1.540385 & 6.65528 & 1.03616 & 5.61912 & accepted \\
\hline 2 & & 1.690715 & 12.64001 & 0.03529 & 12.60472 & accepted \\
\hline 3 & & 2.008267 & 10.49386 & 2.7219 & 7.77196 & accepted \\
\hline 4 & & 1.697877 & 2.10324 & 13.38671 & 11.28347 & accepted \\
\hline 5 & & 1.920368 & 7.75955 & 6.0639 & 1.69565 & accepted \\
\hline 6 & & 1.592346 & 16.15373 & 0.34142 & 15.81231 & not accepted \\
\hline 7 & & 1.664077 & 13.92992 & 6.12777 & 7.80215 & accepted \\
\hline 8 & & 1.800216 & 17.90569 & 1.27517 & 16.63052 & not accepted \\
\hline 9 & & 1.811259 & 3.26368 & 6.30908 & 3.0454 & accepted \\
\hline 10 & & 1.762203 & 8.92376 & 6.58424 & 2.33952 & accepted \\
\hline 11 & & 1.790071 & 4.57233 & 2.43115 & 2.14118 & accepted \\
\hline 12 & & 1.734421 & 18.55746 & 2.04572 & 16.51174 & not accepted \\
\hline
\end{tabular}




\section{ACKNOWLEDGMENT}

The authors would like to thanks Al-Zaytoonah University of Jordan - Amman, Jordan for supporting the scientific research.

\section{REFERENCES}

[1] Jonathan Howell A. and Hilary Buxton, Face Recognition using Radial Basis Function Neural Networks, Proceedings of British Machine Vision Conference, 1996.

[2] Fei Zuo, Embedded Face Recognition Using Cascaded Structures, Thesis, Technische Univ Eindhoven, China, 2006.

[3] Anissa Bouzalmat, et. al, Face Recognition Using Neural Network Based Fourier Gabor Filters \& Random Projection, International Journal of Computer Science and Security (IJCSS), vol.5, issue.3, pp.376- 386, 2011.

[4] Turk M. and Pentland A., Eigenfaces for recognition, Journal of Cognitive Neuroscience, vol.3, pp.71-86, 1991.

[5] Pushpaja V. Saudagare and D.S. Chaudhari, Facial Expression Recognition using Neural Network -An Overview, International Journal of Soft Computing and Engineering, vol.2, issue.1, pp.224-227, March 2012.

[6] Yao-Jiunn Chen, Yen-Chun Lin, Simple Face-detection Algorithm Based on Minimum Facial Features, The 33rd Annual Conference of the IEEE Industrial Electronics Society (IECON) 5-8Nov 2007, Taiwan, pp.455-460.

[7] Sanjay Kr. Singh, et. al, A Robust Skin Color Based Face Detection Algorithm, Tamkang Journal of Science and Engineering, Vol. 6, No. 4, pp. 227-234, 2003.

[8] Abdenour Hadid, Matti Pietik"ainen and Timo Ahone, A Discriminative Feature Space for Detecting and Recognizing Face, ACM, 2004.

[9] Elise Arnaud, et. Al, A Robust And Automa Tic F Ace Tracker Dedica Ted To Broadcast Videos, IEEE international conference on image processing, 2005.

[10] Zhonglong Z., Jie Y. and Yitan Z., Face Detection and Recognition using Colour Sequential Images, Journal of Research and Practice in Information Technology, Vol. 38, No. 2, pp.135-149, May 2006.

[11] E. Hjelmas and B. K. Low, Face Detection: A Survey, Computer Vision and Image Understanding, vol.83, pp. 236-274, 2001.

[12] Yongzhong Lu, Jingli Zhou, Shengsheng Yu, A Survey Of Face Detection, Extraction And Recognition, Computing and Informatics, Vol. 22, pp.163-195, 2003

[13] Ming-Hsuan Yan, et. Al, Detecting Faces in Images: A Survey, IEEE Transactions on Pattern Analysis And Machine Intelligence, Vol. 24, No. 1, pp.34-58, Jan2002.

[14] W. Zhao, R. Chellappa, A. Rosenfeld and P. Phillips. Face recognition: A literature survey, ACM Computing Surveys, Vol.35, No.4, Dec2003, pp. 399-458.

[15] Cha Zhang and Zhengyou Zhang, A Survey of Recent Advances in Face Detection June 2010, Technical Report, MSR-TR-2010-66, Microsoft Research, Microsoft Corporation One Microsoft Way, Redmond, WA 98052.

[16] Rob McCready, Real-Time Face Detection on a Configurable Hardware Platform, Master of Applied Science thesis in the Graduate Department of Electrical and Computer Engineering, University of Toronto, 2000.

[17] Paul V. and Michael J. Jones. Robust real-time object detection, Proc. of IEEE Workshop on Statistical and Computational Theories of Vision, 2001.
[18] G. Shakhnarovich, et al. A Unified Learning Framework for Real Time Face Detection and Classification, IEEE, Proceedings of the Fifth IEEE International Conference on Automatic Face and Gesture Recognition (FGRí02), 2002.

[19] Paul V. and Michael J., Robust Real-Time Face Detection, International Journal of Computer Vision, 57(2), 137-154, 2004, Kluwer Academic Publishers. Netherlands.

[20] Mustafah, et. al, Real-Time Face Detection and Tracking for High Resolution Smart Camera System, Digital Image Computing Techniques and Apps, pp:387-393, 2007.

[21] Zhiwei Zhu and Qiang Ji, Robust real-time eye detection and tracking under variable lighting conditions and various face orientations, Computer Vision and Image Understanding, vol.98, pp:124-154, 2005.

[22] Michalis Zervos, Multi-camera face detection and recognition applied to people tracking, Master Thesis, January 2013, Education and Lifelong Learning of the European Social Fund (ESF) and the NSRF 2007-2013.

[23] Mohammad A. Alia, Abdelfatah Aref Tamimi and Omaima N. A. AL-Allaf, Integrated System for Monitoring and Recognizing Students During Class Session, AIRCC's : International Journal of Multimedia \& Its Applications (IJMA), Vol.5, No.6, Dec2013, pp:45-52.

[24] Vijay Lakshmi and PatilKulakarni, Segmentation Algorithm for Multiple Face Detection in Color Images with Skin Tone Regions using Color Spaces and Edge Detection Techniques, I. J. of Computer Theory and Eng., Vol. 2, No. 4, pp.552-558, August, 2010.

[25] V.K. Narendira Kumar and B. Srinivasan, Internet Passport Authentication System Using Multiple Biometric Identification Technology, I.J. Information Technology and Computer Science, 2013, 03, 79-89, 10.5815/ijitcs

[26] Arindam Kar, A Face Recognition Approach Based on Entropy Estimate of the Nonlinear DCT Features in the Logarithm Domain Together with Kernel Entropy Component Analysis, I.J. Information Technology and Computer Science, 09, pp:31-42, August 2013, MECS.

[27] Prakash C. S., et. al. Fingerprints, Iris and DNA Features based Multimodal Systems: A Review, I.J. Information Technology and Computer Science, 2013, 02, pp:88-111.

[28] Bell, John L. The Art of the Intelligible: An Elementary Survey of Mathematics in its Conceptual Development. 1999, Kluwer. ISBN 0-7923-5972-0.

[29] Euclid. Translated by Johan Ludvig Heiberg with an introduction and commentary by Sir Thomas L. Heath, ed. 1956, The Elements (3vols.) (I and II)

[30] Heath, Sir Thomas. The Theorem of Pythagoras. A History of Greek Mathematics (Vol.2., 1921) (Dover Publications, Inc. (1981) ed.). Clarendon Press, Oxford. pp:144, pp:321323, ISBN 0-486-24073-8.

[31] Sterbenz P., Pat H., 1927. Floating-Point Computation, 1927, Prentice-Hall, 1973.

[32] Hazewinkel, Michiel, ed., "Cosine theorem", Encyclopedia of Mathematics, Springer, 2001, ISBN 978 -1-55608-0104

[33] Omaima N. A. AL-Allaf, Abdelfatah Aref Tamimi and Mohammad A. Alia, Face Recognition System Based on Different Artificial Neural Networks Models and Training Algorithms, International Journal of Advanced Computer Science and Application, Vol.4, Issue.6, pp:40-47,Jun 2013.

[34] Abdelfatah Tamimi, Omaima AL-Allaf and Mohammad Alia, Eigen Faces and Principle Component Analysis for Face Recognition Systems: A Comparative Study, International Journal of Computers \& Technology (IJCT), Vol.14, N4., pp:5650-5660, 28Feb2015. 


\section{Authors' Profiles}

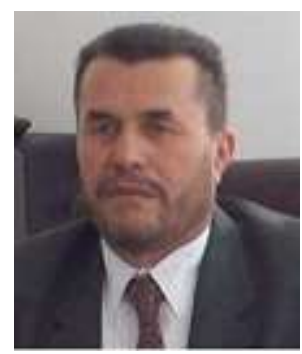

Dr. Abdelfatah A. Tamimi has been a member of the Faculty of Science and Information Technology at AlZaytoonah University since 1996, where he held different positions including the Dean of the Faculty and the Chair of the Department of Computer Science. He received his $\mathrm{Ph} . \mathrm{D}$. in computer science from the City University of New York, NY, USA; his Master's degree in computer science from Montclair State University, NJ, USA; and his Bachelor's degree in mathematics from Jordan University, Amman, Jordan. In addition to his research and teaching experience, he has a 15 year experience in information technology design, development and implementation in United States companies.

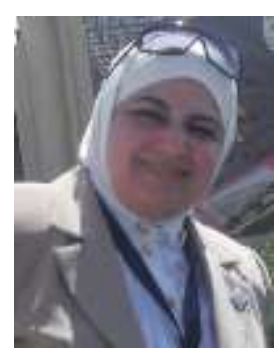

Dr. Omaima N. A. Al-Allaf is an Assistant Professor at Basic Sciences department, Faculty of Sciences and IT, AL-Zaytoonah University of Jordan, Amman-Jordan (from 15 Feb. 2009). She received her B.Sc. in Computer Science from Dept. of CS, Faculty of Sciences, University of Mosul (19901994), and received her M.Sc. from Dept. of CS, Faculty of Computer \& Mathematical Sciences, University of Mosul (1997-1999), Mosul, Iraq. She worked as Teacher B in Applied Science University (Private)-Amman-Jordan from 2000 to 2004. She received her PhD in CIS from the Department of CIS, AABFSAmman-Jordan (2004-2008). During 2004-2008, she worked as a part time lecturer in the AABFS. Her research interests are in the fields of: Artificial Intelligence, Artificial Neural Networks, Image Processing, Web Engineering, and Software Process Models.

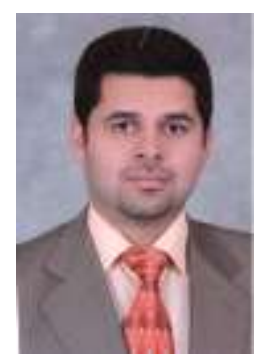

Dr. Mohammad Alia is an associate professor at the Computer Information Systems department, Faculty of Science and Information Technology, AlZaytoonah University of Jordan. He received the B.Sc. degree in Computer Science from Al-Zaytoonah University, Jordan, in 2000. He obtained his Ph.D. degree in Computer Science from University Science of Malaysia, in 2008. During 2000 until 2004, he worked at Al-Zaytoonah University of Jordan as an instructor of Computer Sciences and Information Technology. Then, he worked as a lecturer at AlQuds University in Saudi Arabia from 2004 to 2005. Currently he is working as the Chair of Computer Information Systems dept. at Al Zaytoonah University of Jordan. His research interests are in the field of Cryptography and Network Security.

How to cite this paper: Abdelfatah Aref Tamimi, Omaima N. A. AL-Allaf, Mohammad A. Alia,"Real-Time Group FaceDetection for an Intelligent Class-Attendance System", International Journal of Information Technology and Computer Science(IJITCS), vol.7, no.6, pp.66-73, 2015. DOI: 10.5815/ijitcs.2015.06.09 